\title{
Antioxidant Therapies for Neurodegenerative Diseases: Mechanisms, Current Trends, and Perspectives
}

\author{
Daniel Pens Gelain, ${ }^{1}$ Guilherme Antonio Behr, ${ }^{2}$ \\ Ramatis Birnfeld de Oliveira, ${ }^{3}$ and Madia Trujillo ${ }^{4}$ \\ ${ }^{1}$ Department of Biochemistry, Center for Oxidative Stress Research, Federal University of Rio Grande do Sul (UFRGS), \\ 90035-003 Porto Alegre, RS, Brazil \\ ${ }^{2}$ Department of Psychiatry and Behavioural Neurosciences, McMaster University, Hamilton, ON, Canada L85 4K1 \\ ${ }^{3}$ Experimental \& Translational Respiratory Group, University of Newcastle, Newsastle, NSW 2308, Australia \\ ${ }^{4}$ Department of Biochemistry, Center for Free Radical and Biomedical Research, School of Medicine, Universidad de la República, \\ 11800 Montevideo, Uruguay
}

Correspondence should be addressed to Daniel Pens Gelain, dgelain@yahoo.com.br

Received 7 November 2012; Accepted 7 November 2012

Copyright ( $\odot 2012$ Daniel Pens Gelain et al. This is an open access article distributed under the Creative Commons Attribution License, which permits unrestricted use, distribution, and reproduction in any medium, provided the original work is properly cited.

Over the last decades, the involvement of free radicals and oxidative stress in the molecular mechanisms related to ageing, cancer, atherosclerosis, neurodegenerative disorders, diabetes, and inflammatory diseases became increasingly clear. As a consequence, the interest on the potential therapeutic applications of antioxidants has also increased. Naturally occurring antioxidants as well as modified or synthetic compounds have been screened for biological activities that could be useful in the development of new therapies for diverse conditions. Research using in vitro (cell culture and chemical free radical-generating systems), in vivo (animal models), and in silico models as well as epidemiologic and different types of clinical studies have been conducted by a great number of researchers worldwide, in order to evaluate the possible therapeutic properties of compounds with antioxidant activity. However, despite the massive accumulation of evidence confirming the role of oxidants in several pathological conditions (especially cancer), the absolute majority of antioxidants developed for clinical use has been proven ineffective.

In fact, a considerable number of compounds intended to act as antioxidants in developing therapies were actually reported to induce acute or long-term toxic effects, including oxidative stress itself. One of the best documented examples is the case of vitamin A, which was widely regarded as an essential antioxidant component of the diet during the 1980 decade. A large clinical trial in the early 1990s The
Beta-Carotene and Retinol Efficacy Trial (CARET) evaluated the effect of oral intake of beta-carotene and retinol in 18,314 men and women at high risk for lung cancer, but it had to be discontinued due to increased incidence of lung cancer, cardiovascular disease, and mortality. Following this study, several papers demonstrated that according the concentration, type of administration, and other factors, vitamin A may act as a strong pro-oxidant to living cells. Presently, some authors believe that even pro-oxidant agents or processes, which were earlier recommended to be avoided, may be important to maintain a healthy status, as low/mild exposure to free radicals would help cells to maintain an effective antioxidant defense system through physiological mechanisms, in a process that could be called "oxidative stress preconditioning." Thus, it is clear that antioxidants and antioxidant-based therapies must be cautiously addressed by researchers in the field of redox biology.

One of this special issue papers investigates the effect of the antioxidants trolox (hydrophilic analogue of alphatocopherol) and dithiothreitol (DTT) on mouse locus coeruleus neurons. This study demonstrates that pacemaker currents and firing rates were heterogeneously modulated in half of the neuronal population accessed, leading to the hypothesis that these neuronal populations could be the first ones affected in neurological disorders involving imbalance in the oxidant/antioxidant content. Another paper of this special issue presents an investigation on the potential of an 
ancient traditional medicine used in China, the pine pollen, as a controller of cellular senescence through reduction of oxidative stress and free radical-related formation of advanced glycation endproducts (AGEs), which are relevant inducers of chronic inflammation and neurodegenerative processes. Another paper in this issue demonstrates that the redox impairment caused in hippocampus by the molecular constituent of amyloid plaques, the amyloid-beta peptide $(\mathrm{A} \beta)$, is a result of increased $\mathrm{A} \beta$ cellular internalization and deposition to mitochondria, and melatonin is an important endogenous protector in this context. Another article is a review on the possible mechanisms involved in the antioxidant action of S-allylcysteine and old garlic extract, which is enriched in S-allylcysteine, on different biological systems, evaluating the potential clinical and therapeutic applications of these products.

Another paper of this special issue reviews the clinical literature on oxidative and antioxidant effects associated with antidepressant agents and discusses their potential effects in the treatment of major depressive disorder. This interesting analysis suggests that augmentation of antioxidant defenses may be one of the mechanisms underlying the neuroprotective effects of antidepressants in major depressive disorder therapies. Another work published in this special issue addresses an innovative topic, reviewing the recent advances in therapies for neurodegenerative diseases that are based on glutathione (GSH) delivery systems. Glutathione is regarded as one of the major intracellular antioxidants, but its limited stability and bioavailability pose serious difficulties in the development of GSH-based therapies for clinical application. Another work in this issue addresses the redox and transcriptional effects of gases used in medicine (such as carbon monoxide, hydrogen sulphide and hydrogen) on antioxidant enzymes. Finally, another article of this special issue is an updated review on the current state of the use of antioxidants for therapies aimed to Alzheimer's disease. This comprehensive work summarizes many of the aspects that must be taken into account when dealing with antioxidants that exhibit a potential therapeutic application. Many compounds first appear to be promising in animal and in vitro models but fail to show relevant clinical results. Also, the relationship between endogenous antioxidants with disease prevention and/or prevalence revealed to be predictive in some epidemiological and transversal studies but were not observed in other similar works. The reasons accounting for such inconsistency are still not clear but certainly urge both caution and more extensive research on this subject.

Daniel Pens Gelain Guilherme Antonio Behr Ramatis Birnfeld de Oliveira Madia Trujillo 


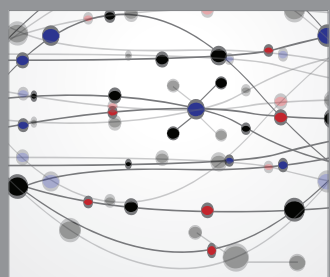

The Scientific World Journal
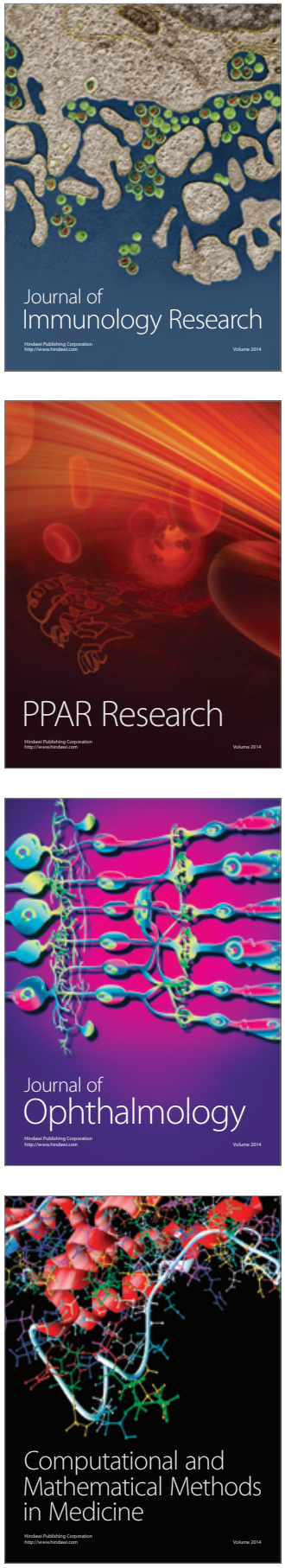

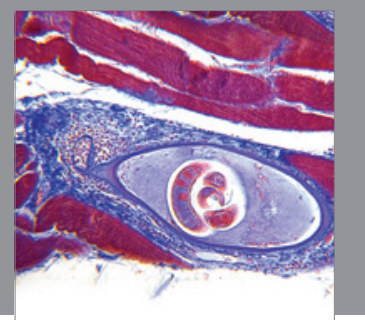

Gastroenterology

Research and Practice
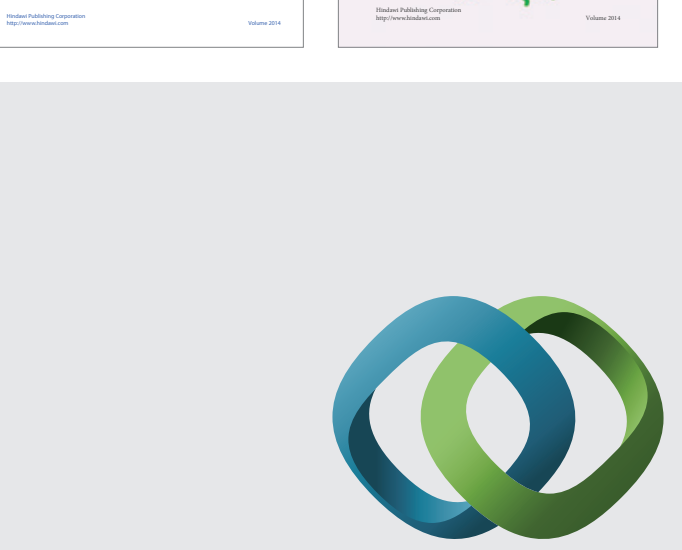

\section{Hindawi}

Submit your manuscripts at

http://www.hindawi.com
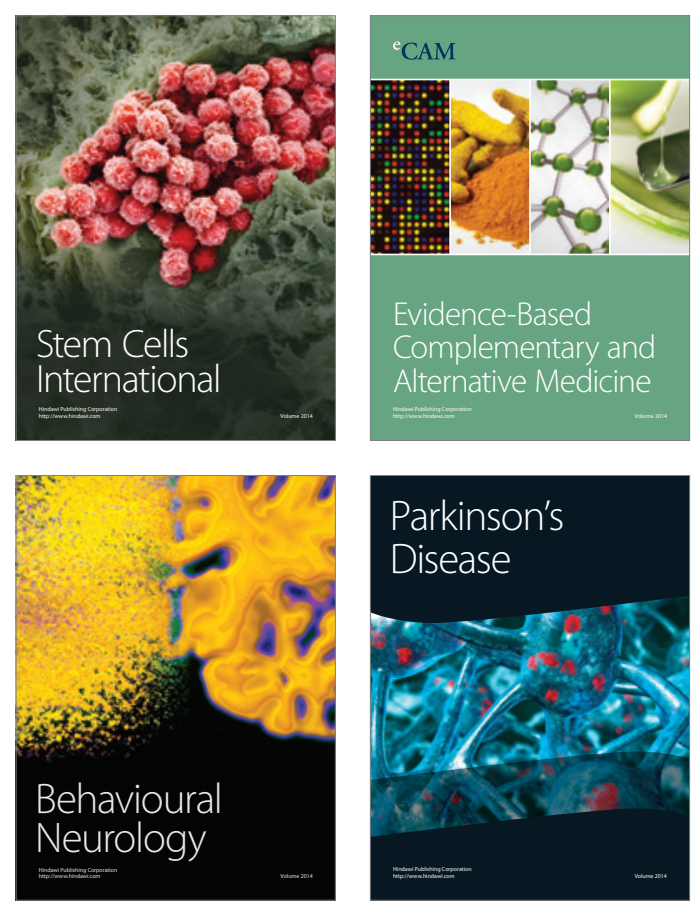

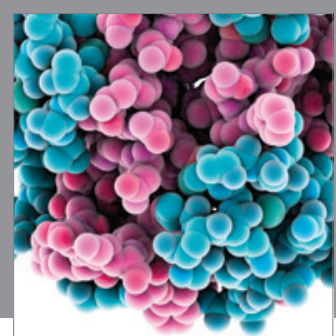

Journal of
Diabetes Research

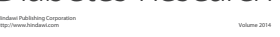

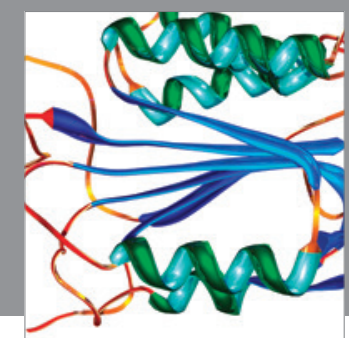

Disease Markers
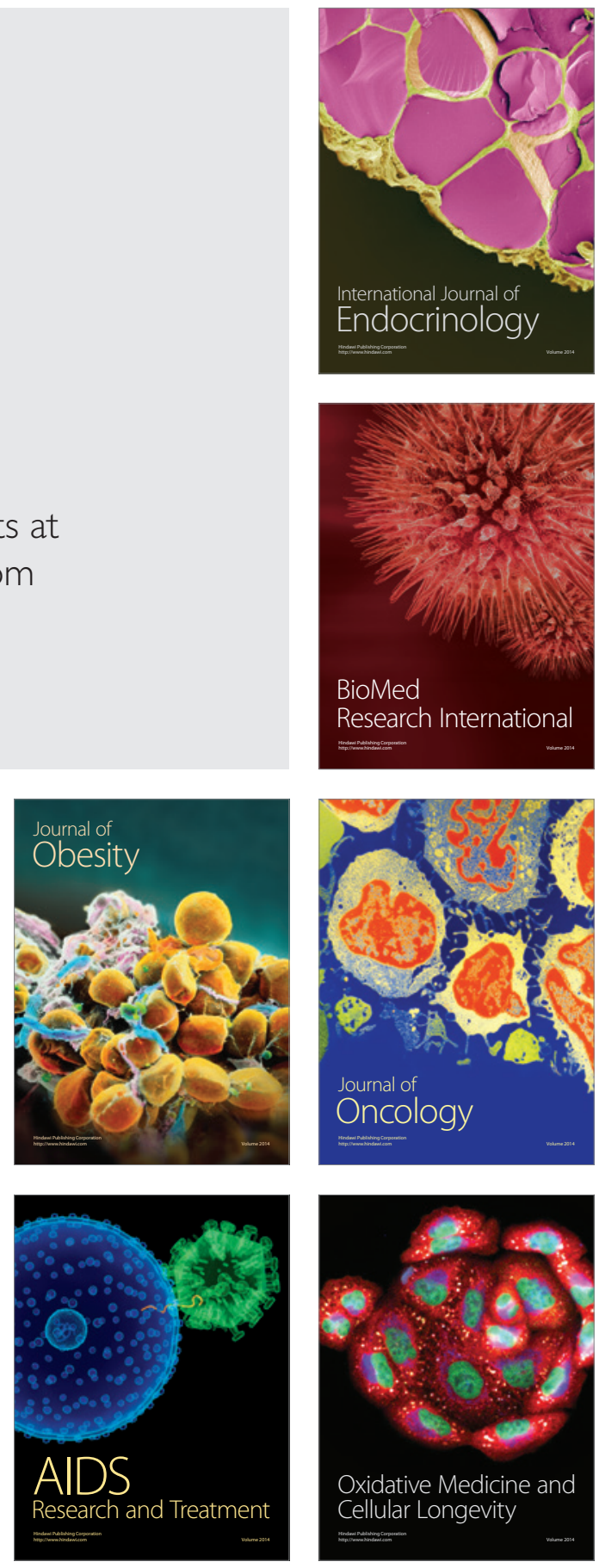